University of Nebraska - Lincoln

DigitalCommons@University of Nebraska - Lincoln

Winter 2021

\title{
An Introduction to Blockchain Technology and Its Application in Libraries
}

ANUBHAW SUMAN, Research Scholar, MGCUB

sumanubhaw@gmail.com

Madhu Patel , Assistant Professor, MGCUB

madhupatel@mgcub.ac.in

Follow this and additional works at: https://digitalcommons.unl.edu/libphilprac

Part of the Archival Science Commons, Cataloging and Metadata Commons, Information Literacy Commons, Scholarly Communication Commons, and the Scholarly Publishing Commons

SUMAN, ANUBHAW , Research Scholar, MGCUB and Patel, Madhu, Assistant Professor, MGCUB, "An Introduction to Blockchain Technology and Its Application in Libraries" (2021). Library Philosophy and Practice (e-journal). 6630.

https://digitalcommons.unl.edu/libphilprac/6630 


\title{
An Introduction to Blockchain Technology and Its Application in Libraries
}

\author{
Anubhaw Kumar Suman \\ Research Scholar (PhD) \\ Department of Library \& Information Science \\ Mahatma Gandhi Central University, Motihari, Bihar- 845401, India \\ Email: sumanubhaw@gmail.com \\ Dr. Madhu Patel \\ Assistant Professor \\ Department of Library \& Information Science \\ Mahatma Gandhi Central University, Motihari, Bihar- 845401, India. \\ Email: madhupatel@mgcub.ac.in
}

\begin{abstract}
The concept of blockchain technological came into existence from the first cryptocurrency, Bitcoin. It is a very new, complex and trusted technology, and researchers are working continuously to find a way to apply this disruptive technology in different areas. This paper discusses a brief concept about the blockchain and the application of blockchain technology in libraries \& information centres to enhance better library and information services in the future, as well as at present. This paper provides a basic understanding of blockchain technology, like what blockchain and blockchain technology is, its features, advantages, types, and how it will be helpful in education and libraries? Blockchain is a very disruptive technology that can reconfigure all aspects of society and its operations. This technology is immutable and distributed, making it difficult for transactions to be changed, duplicated, or faked. The study is an initiative to make library professionals aware of this emerging disruptive technology and guide them in taking some actions for the appropriate application of blockchain technology in libraries.
\end{abstract}

Keywords: Blockchain Technology, Distributed Ledger Technology, Library Records Keeping, Smart Library Services, Library Management System, Digital Rights Management.

Paper Type: Conceptual Paper

\section{Introduction}

Most simply, the blockchain is defined as the decentralized and distributed ledger technology that provides information to be recorded, maintained and shared by a community. It is a new 
type of database having digital records of transactions. No person or entity has control over it, and none of them can go back and erase or change a transaction history. Distributed ledger technology combines transparency, immutability and security for the participants of the network. It is nearly impossible to hack or attack the entire system based on blockchain technology because of the distributed nature of this technology. Thus, blockchain is a tamperproof technology. In blockchain technology, all transactions performed in the past cannot be changed or deleted. So, it provides transparency and immutability to all transactions that happened in the past (Kushwaha \& Singh, 2020). "Unlike the Web or Internet alone, blockchains are distributed, not centralized; open, not hidden; inclusive, not exclusive; immutable, not alterable; and secure. Blockchain gives us unprecedented capabilities to create and trade value in society" (Tapscott \& Tapscott, 2017).

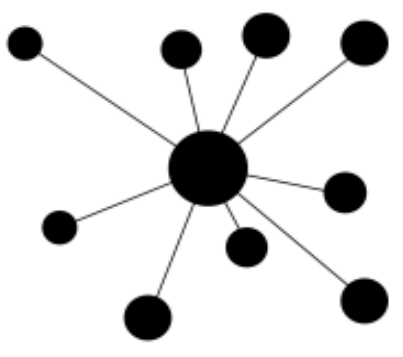

A centralized network.

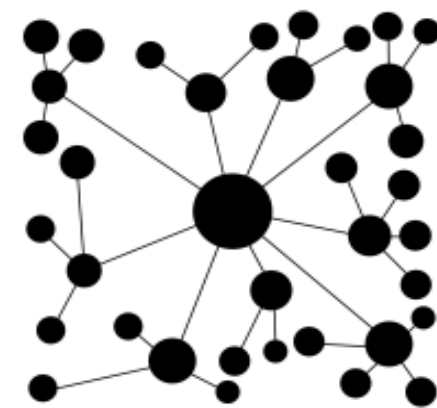

A decentralized network.

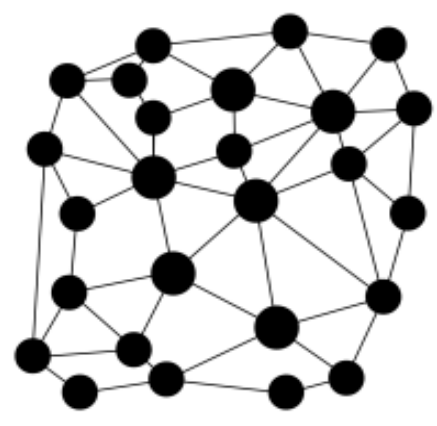

A distributed network.

Fig. 1: A comparison of the topography of centralized, decentralized and distributed networks (Source: Baran, 1964)

Like any other technology revolution, the blockchain technology revolution may also be categorized into three phases as Blockchain 1.0, Blockchain 2.0 and Blockchain 3.0 (Kushwaha $\&$ Singh, 2020). Cryptocurrencies are an application of Blockchain 1.0, which is related to dayto-day digital payment-based systems. Blockchain 2.0 is associated with the whole economic market, where Blockchain technology is used to expand traditional transactions like bonds, stocks, shares and smart contracts. While all those applications which are not included in the scope of Blockchain 1.0 and 2.0 come under Blockchain 3.0, like e-governance, digital health records, digitally vote counting, science, literacy, culture and digital art (Swan, 2015).

However, the applications or usage of blockchain technology are now being explored in many other areas. Currently, industry sectors such as finance, medicine, health science, education and government are making significant investments, investigating the transformational impact of blockchain and searching the future opportunities of implementing this technology in many other sectors, as shown in fig. 2. Blockchain has been found as an excellent solution for many 
issues encountered by the educational community. It can be used for multiple works like issuing, monitoring, validating and sharing the certificates (Kolvenbach, Ruland, \& Grather, 2018). Hoy (2017) suggested that blockchain can also be used as another digital rights management tool. This technology has potential use not only in the financial domain but also in the non-financial domains. Thus, it can be used as a catalyst in developing the library \& library services to accelerate the entire library system globally.

\section{APPLICATIONS OF BLOCKCHAIN}

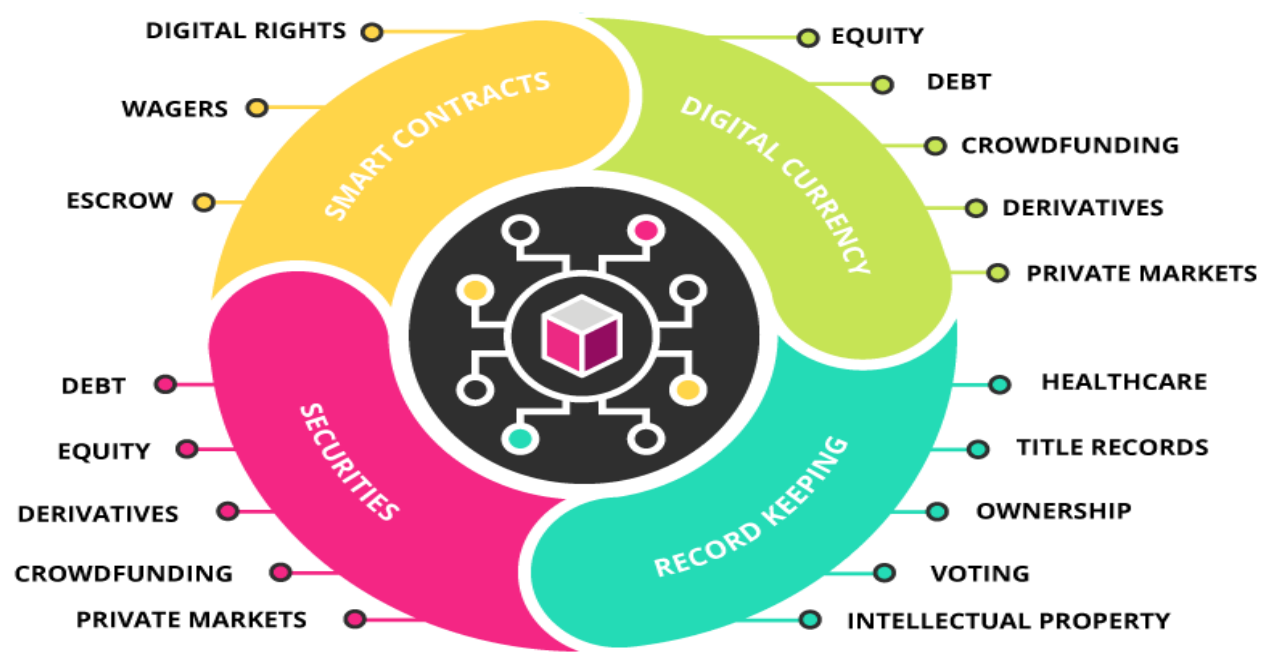

Fig. 2: Application of Blockchain in different areas

(Source: https://www.getsmarter.com/blog/market-trends/blockchains-effect-on-business/)

\subsection{What is Blockchain?}

The name of blockchain came from its structure, i.e. block and chain; individual records, called blocks, are linked together in a series to form the chain. Blockchain is an emerging technology that will change the way we acquire and share information. It is an online global database that anyone, anywhere at any time, with an internet connection, can use. Like traditional databases, it is not owned by any central agency or body like banks and governments. Thus, Hacking or tampering with the entire system based on this technology by faking transactions, documents, and any information becomes nearly impossible. All financial transactions based on this technology are more fast and secure than the traditional ones. Blockchain technology combines many other technologies, like cryptography, peer-to-peer networks, smart contracts, and consensus mechanisms to create a new and unique database. It also logs the time, date, details of participants, and other legal or contractual portions of every transaction. Blockchain is the primary technology behind cryptocurrencies like Bitcoin and Ethereum, making it secure for 
trading digitally by verifying and storing transaction records in a distributed and time stamp manner. The term Bitcoin, a cryptocurrency, was first introduced in 2008 by Satoshi Nakamoto in his work named "Bitcoin: A Peer-to-Peer Electronic Cash System" (Hoy, 2017).

\subsection{How does Blockchain Works?}

In general, Blockchain is a chain of blocks, and a block consists of three things: Data, Hash, and Hash of the previous block. Each block in the chain contains a cryptographic hash of its own and the last block to stay connected in the chain.

A Block is a primary unit of blockchain. In the blockchain, a block is a collection of data or information. The information is added to the block in the blockchain by connecting it with other blocks in chronological order and creating a chain of blocks linked together. Thus, it forms a chronological database of transactions that is shared with multiple nodes, i.e. computers or servers, in a network. The unique number added to a hashed or encrypted block called Nonce that can be used only once is selected by the miners to solve a cryptographic puzzle for generating the next block in the chain. It is known as Proof of Work.

A Hash is a unique alphanumeric identifying code or number generated when any transaction happens in the blockchain. Hash is based on data of its own, a hash of the previous one, and its timestamp (Peck, 2017).

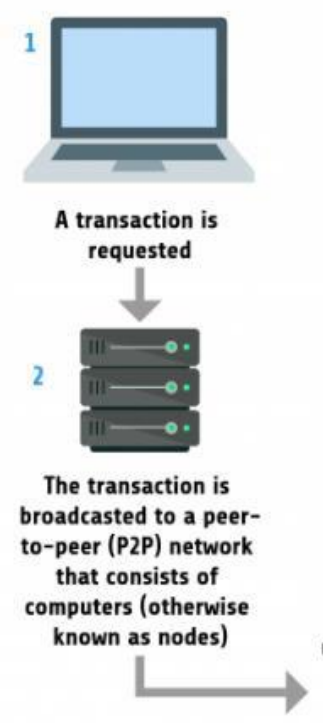

How
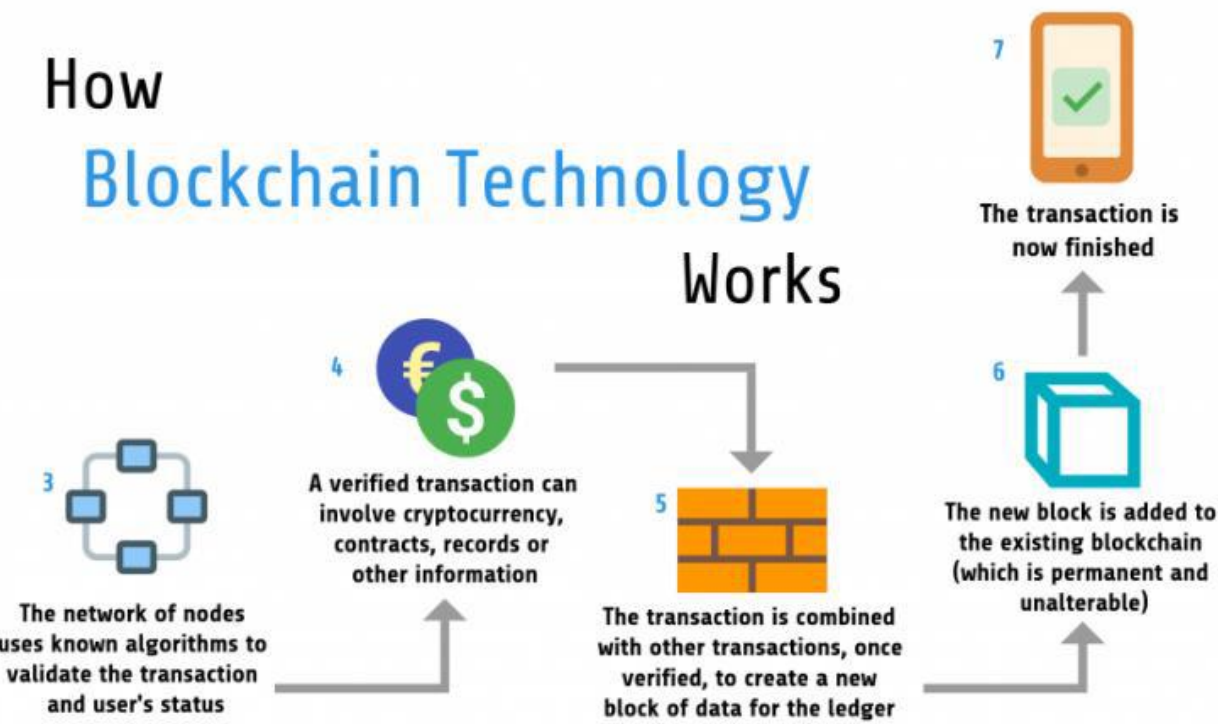

Fig. 3: How Blockchain Technology Works (Source:

https://medium.com/@ipspecialist/how-blockchain-technology-works-e6109c033034)

When a transaction (fig. 3) happens in the blockchain, that transaction is recorded in a block, and that block must be validated before adding it into the chain. The authenticity of a block 
must be verified through a consensus algorithm in which the majority of nodes (clients or servers) and the nodes having the highest stack in the chain of the distributed network must validate the block before adding to it in a chain. After the validation of the block, a unique, identifying code, i.e. a hash, is generated. By doing this, we do not need any third-party interference to validate or to do transactions.

Blocks can be recognized by their block number or block height and block header hash. The data in the blocks is detected through a computerized algorithm known as the hash function. This function locks the data to be seen by the participants in the Blockchain and makes the data immutable. Every block has its own hash function. In Blockchain, the data is recorded for permanent and will not be changed. A small change in it generates a new block in the chain. Blockchain works like a digital notary with time-stamps to avoid tampering with any information.

\section{Review of Literature}

Many studies of the recent past have been done to get the basic idea and clarity about the concept of blockchain, and some of those are as follow:

Verma (2021) carried out a study on the amalgamation of Blockchain Technology with Knowledge Management System and concluded that the Blockchain-based library would become the primary book management method for institutions or organizations in the future. The library management system would be Blockchain-based record-keeping of acquisition and circulation of books, fee or the fine collection and stock verification of library documents.

ALA (2021) stated that Similar to artificial intelligence and other disruptive technologies, blockchain has the capability to change the functional and structural framework of many of the financial as well as non-financial institutions that are built by humans (like banks $\&$ insurance companies etc.) and to dislocate the administrative workers that nurture those institutions. Blockchain can facilitate more secured and trusted certification, and its use could expand across formal and informal learning in academic, public, and special libraries.

In their article, Tenorio-Fornes \& et al. (2019) mentioned that current methods of scientific publication and peer review raise concerns around performance, fairness, cost, quality, and accuracy. He also described the demerits of the open access movement that the open access system has been unable to fulfil all its promises. The present model is publisher centred, where publishers can impose policies and concentrate profits at any time. Using innovative distributed technologies like blockchain, he proposes a decentralised system for publication in the area of 
open science. The plan may provide (i) a distributed reviewer reputation system, (ii) an Open Access by-design infrastructure, and (iii) transparent governance processes.

Chen \& et al. (2018) discussed the current use and future implications of blockchain technology in academia, library and many other sectors or industries.

Coghill (2018) examines the potential uses for Bitcoin and Blockchain technology in libraries and the possibility of blockchain for transferring and payment the funds from libraries to vendors and maintaining electronic receipts as digital evidence.

Hoy (2017), in his study, suggested that the gathering, preserving and sharing of authoritative information can quickly and easily be done with the help of blockchain technology. It can also be a solution to prevent copyright issues as it produces a unique, verifiable record that is difficult to reproduce by an outsider. Further, he suggested the technology for creating a timestamped, verifiable version of journal articles. He also pointed out the negative side of blockchain as technical complexity and environmental unsustainability.

Debbie (2017) explored the law libraries have a further possibility for authenticating primary law documents and also explore legal aspects of smart contracts as a medium for paying for electronic resources.

\section{Core Features of Blockchain Technology}

i. In the blockchain, Blocks can be written and read by certain and authentic participants, and all entries are permanent, transparent, and searchable.

ii. All transactions in blockchain technology are recorded in chronological order on a continuously growing and permanent database.

iii. Blockchain is a tamper-proof technology. It is nearly impossible to hack or attack the entire system based on blockchain technology because of its distributed nature.

iv. In this technology, data is replicated and stored across the system over a peer-to-peer network.

v. It facilitates the peer-to-peer transfer of value without a central intermediary like- a bank or any financial institution.

vi. In the blockchain, digital signatures and cryptography are used to secure the transaction.

vii. In blockchain technology tokenization process is followed where the value of an asset (physical or digital) is converted into digital tokens that are recorded and shared via blockchain.

viii. It is significantly faster \& cost-efficient technology than conventional methods by eliminating intermediaries and replacing remaining manual processes. 


\section{Some Key Advantages of Blockchain Technology}

- Real-Time Transaction Settlement

- More Security \& Transparency

- No Third-Party Involvement

- Durability \& Reliability

- Immutability

- Cost Saving

- User Pseudonymity

\section{Types of Blockchain Technology}

Blockchain technology is primarily of two types; Public blockchain and Private blockchain, but two other forms of blockchain also exists; Hybrid blockchain and Consortium or Federated blockchain. The brief discussion on all types of blockchain, as mentioned above, are as follow:

5.1 Public Blockchain: Public blockchains are such blockchains that are accessible publicly. It is a type of blockchain, "for the people, by the people, and of the people" (Sanjay \& Nabi, 2020). A public blockchain is permission-less distributed ledger technology where anyone (node or end-user) may join and do the transactions. They do not require unique authentication (login with user id \& password) to access, read, write, and update the blockchain. This technology is used in cryptocurrencies like Bitcoin, Ethereum \& Litecoin etc.

5.2 Private Blockchain: A private blockchain is permission-able or restrictive blockchain technology, functional only in a closed network. This blockchain is usually used within an organization or firm where only authentic members can access and do the transactions in the network. Participants of this network require unique authentication or authorization (login with user id \& password) to access, read, write and update in the blockchain. Such blockchains are generally less-trusted than public blockchains. A private blockchain can be used in voting, digital identity \& supply chain management etc.

5.3 Hybrid Blockchain: A Hybrid blockchain is a combination of both Public and Private blockchains. Such blockchain uses the features of both types of blockchain. "A transaction in a private network of a hybrid blockchain is usually verified within the same network, but participants may also release it in the public blockchain to get verified." The organization that neither wants to deploy a private blockchain nor public blockchain can simply deploy this blockchain. Example: Dragonchain. 
5.4 Consortium Blockchain: Consortium blockchain is also known as Federated blockchain. This blockchain network is managed by a set of organizations or nodes rather than a centralized or decentralized network. It is an ideal solution that requires collaboration across the board. For instance, supply chain, food, and medicine would need an alliance across brands. Example: Energy Web Foundation, Ripple etc.

\section{Applications of Blockchain in Libraries}

Blockchain technology has encouraged librarians or library professionals to adopt this technology at various levels of library operations. Libraries in the western world started to adopt this technology in a speedy manner. Some of them explored how to use this technology to build an enhanced metadata system for libraries, protect digital first sale rights, host digital peer-to-peer (P2P) sharing, etc. ("Ways to Use Blockchain in Libraries," 2017). So, some of the blockchain technology applications in libraries are as discussed below:

6.1 Publishing E-Books \& Protect Digital First Sale Right: At present, to use the eBook, we have third party interference, and Libraries or users are forced to pay for the complete package whether they use it or not. Also, the author does not get complete control over its creation. But, blockchain can help us to overcome this problem. Authors can create a smart contract, where they can mention their own terms and conditions for the use of their book. Further, a smart contract creates an Etherum network where anyone can purchase a book through the book token. Publica, a platform where anyone can publish their book, has developed a new feature called crowdfunding, where the creator can pre-sell their token so that one can be able to raise funds for some initial payments.

6.2 Research Institutions Network on Blockchain: A private blockchain network can use to share research amongst all the research institutions like IITs, NITs, IIITs and other such types of institutions. This network can use to publish grey literature, lab papers, test reports and documents that are significant in carrying out the research but not in a proper format to publish as an article or a book. Thus, everyone in blockchain can access that research rather than doing parallel research and may also use that crucial information in their research. In this system, blockchain participants do not need to care about digital rights, counterfeiting of the data, and no one can alter the data (Sanjay \& Hasan, 2020).

6.3 Academic or Scholarly Publishing: As we all know, the current model of publishing the research paper is publisher centred where the publisher has all the rights, and the author has to stay behind the curtain, always; for example, everybody knows about the famous journals, 
but nobody cares about the authors. But by using the Orvium platform, which uses blockchain technology, anyone can register themselves by using Orvium GUI, or the scholars can use their ORCiD ID for the registration process. Therefore, when the authors publish something on Orvium, they receive tokens, and these tokens can be used to pay for the peer review process, and the review process can be blind or open (Gul \& Shohar, 2019). Blockchain could make a digital asset purchase of the research papers easier by assigning every research paper a Bitcoin address (QR code) instead of requiring users to log in to publisher websites. In academic publishing, blockchain technology can also be used for plagiarism detection and avoidance of plagiarism by auto-citation (an Ethereum smart contract that does a literature search and automatically cites all the related literature). Thus, it would be an excellent time-saver for researchers (Swan, 2015).

6.4 Digital Rights Management: Blockchain technology can also be used as Digital Rights Management (DRM) tool for e-resources available in libraries. Nowadays, most libraries and publishers facing a problem of reproducibility of e-resources. But this problem can be solved if blockchain technology is tied up with e-resources. It will make the e-resources to be identified, controlled and transferred uniquely (Griffey, 2016).

6.5 User to User \& Library to User Book Loan: The other most crucial blockchain application in the library is borrowing the book from the library user or another library without coming to the library. With the help of Libchain, a Distributed Library Management System based on blockchain technology, patrons can provide the library books directly to other library users without bringing the book or any reading document back to the library. But the only condition is the patron should be a valid member of the library. Similarly, this system supports Inter-Library Loan (ILL) procedures or services among the member libraries of the blockchain network. "LibChain aims to extend popular library services and creates a library ecosystem where the patron gets their desired service efficiently, comfortably and securely" (Oyelude, 2019). In the blockchain, authentic library users can control their circulation records and manage their browsing \& borrowing histories with the approval of library staff in the chain.

\section{Conclusion:}

Blockchain is an emerging technology that provides librarians and library professionals a unique opportunity to promote and facilitate collaboration among the libraries and users to enhance user-centred library services. It offers library professionals an opportunity to identify, collect, customize, organize, preserve, and deliver information products and services to the 
end-users on-demand or in anticipation (Ragavan et al., (n.d.). Librarians or library professionals will be highly benefited if they use this technology to order books from vendors, pay vendors, subscribe to journals and manuscripts from publishers, exchange sensitive and confidential records, share authoritative information with other parties of the library network. It may also prevent copyright issues, protect digital first sale rights, support scholarly publishing and Digital Rights Management, etc. In fast-developing countries like India, where the growth rate of library culture is slow, it is need of the day to explore the latest trending technology like blockchain for the radical improvement of library and library services, and there is no doubt that the blockchain is one of such technology, which can bring the radical changes in library services.

\section{References:}

Swan, M. (2015). Blockchain: Blueprint for a new economy. (T. McGovern, Ed.). Beijing: O'Reilly Media. Retrieved from https://silo.pub/blockchain-blueprint-for-a-neweconomy.html

Kushwaha, A. K., \& Singh, A. P. (2020). Connecting blockchain technology with libraries: opportunities and risks. Journal of Indian Library Association, 56(3), 12-19. Retrieved from https://www.ilaindia.net/jila/index.php/jila/article/view/408; https://ilaindia.net/jila/index.php/jila/article/viewFile/408/209 (Accessed August 10, 2021)

Grather, W., Kolvenbach, S., Ruland, R., Schutte, J., Torres, C. F., \& Wendland, F. (2018). Blockchain for Education: Lifelong Learning Passport. Proceedings of $1^{\text {st }}$ ERCIM Blockchain Workshop 2018. European Society for Socially Embedded Technologies (EUSSET). https://doi.org/10.18420/blockchain2018

Hoy (M B). (2017). An introduction to the blockchain and its implications for libraries and medicine. Medical Reference Services Quarterly. 36(3): 273-279.

https://doi.org/10.1080/02763869.2017.1332261

IoT powered by blockchain: How blockchains facilitate the application of digital twins in IoT:https://www.academia.edu/40165830/IoT_powered_by_Blockchain_How_Blockchains_f acilitate the application_of_digital_twins_in_IoT (Accessed August 10, 2021).

Sanjay \& Hasan, N. (2020). Blockchain technology and its application in libraries. Library Herald, 58(4), 118-125. (ISSN-Print-0024-2292, Online- 0976-2469).

https://doi.org/10.5958/0976-2469.2020.00030.10;

https://www.researchgate.net/publication/348602358_Blockchain_Technology_and_its_Appl ication_in_Libraries

Verma, M. (2021). Amalgamation of blockchain technology and knowledge management system to fetch an enhanced system in library. International Journal of Innovative Research in Technology, 7(11), 474-477. Retrieved from 
https://www.researchgate.net/publication/351287033_Amalgamation_of_Blockchain_Techno logy_and_Knowledge_Management_System_to_fetch_an_enhanced_system_in_Library

Ways to Use Blockchain in Libraries. (2017)., Accessed September 05, 2021, from https://ischoolblogs.sjsu.edu/blockchains/blockchains-applied/applications/

ALA. (2021). Blockchain., Accessed September 06, 2021, from https://www.ala.org/tools/future/trends/blockchain

Griffey, J. (2016). Blockchain and Intellectual Property @ Internet Librarian 2016. Retrieved May 21, 2021, from https://jasongriffey.net/wp/2016/10/21/blockchainintellectual-propertyinternetlibrarian-2016/

Debbie, G. (2017). Law and the Blockchain by Debbie Ginsberg. Retrieved June 07, 2021, from https://ischoolblogs.sjsu.edu/blockchains/law-and-the-blockchain-bydebbie-ginsberg/

Chen, G., Xu, B., Lu, M., \& Chen, N.-S. (2018). Exploring blockchain technology and its potential applications for education. Smart Learning Environments, 5(1), 1-10.

https://doi.org/10.1186/s40561-017-0050-x

Coghill, J. G. (2018). Blockchain and its implications for libraries. Journal of Electronic Resources in Medical Libraries, 15(2), 66-70.

https://doi.org/10.1080/15424065.2018.1483218

Lemieux, V. L., Hofman, D., Batista, D., \& Joo, A. (2019). Blockchain technology \& recordkeeping. ARMA International Educational Foundation. Retrieved from www.armaedfoundation.org

Tenorio-fornes (A), et al. (2019). Towards a decentralized process for scientific publication and peer review using blockchain and IPFS. 52nd Hawaii International Conference on System Sciences, 2019:

https://scholarspace.manoa.hawaii.edu/bitstream/10125/59901/1/0461.pdf

LibChain: Distributed library management system based on the blockchain technology: https://www.atositchallenge.net/wp-content/uploads/2016/11/LibChain-Atos-IT-Challenge2017.pdf (Accessed September 01, 2021).

CoinSutra. Agrawal Harsh //CoinSutra. Accessed March 10, 2021, from https://coinsutra.com/different-types-blockchains/

Iredale, Gwyneth (2021). What are the different types of blockchain technology? Accessed August 10, 2021, from https://101blockchains.com/types-of-blockchain/

P. Baran, "On Distributed Communications Networks," IEEE Transactions on Communications Systems 12, no. 1 (March 1964): 1-9, https://doi.org/10.1109/TCOM.1964.1088883

Ragavan, S. S., Konnur, P. V., \& Prabu, R. (n.d.). Emerging technologies for library and information services in the digital era. Retrieved from 
http://14.139.186.108/jspui/bitstream/123456789/2460/1/emrgingtechnologies\%2C orisa.doc.pdf

Peck (M E) (2017). Blockchains: How they work and why they will change the world. IEEE Spectrum: https://spectrum.ieee.org/computing/networks/blockchains-how-they-work-andwhy-theyll-change. -the-world (Accessed June 10, 2021).

Oyelude (A A) (2019). What's trending in blockchain technology and its potential uses in libraries. Library Hi Tech News. 36(9): 17-18.

Gul (S) and Shohar (B). (2019). Smart libraries: An emerging and innovative technological habitat of 21st century. The Electronic Library. 37(5): 764-783.

Chingath (Vysakh) and babu (H. Rajendra). (2020). Advantage of blockchain technology for the Libraries:

https://www.researchgate.net/publication/341725555_Advantage_Blockchain_Technology_f or_the_Libraries (Accessed August 16, 2021).

Tapscott, D., \& Tapscott, A. (2017); Realizing the potential of blockchain; World Economic Forum. http://www3.weforum.org/docs/WEF_Realizing_Potential_Blockchain.pdf. Retrieved August 15, 2021, from http://www3.weforum.org (Accessed August 15, 2021)

Accelerating Scientific Publishing. Orvium: https://orvium.io (Accessed August 10, 2021).

Investopedia: https://www.investopedia.com/about-us (Accessed August 10, 2021).

DataFlair. https://data-flair.training/blogs/types-of-blockchain/ (Accessed August 10, 2021).

Publica wants to free publishers and authors from Amazon using the blockchain: https://www.thebookseller.com/futurebook/publica-wants-break-publishers-and-authors-outamazon-stranglehold-using-blockchain: (Accessed August 28, 2021).

Pupuweb; 101 Blockchain Cheat Sheet, Jargon, Glossary and Terminology. https://pupuweb.com/blockchain-bitcoin-cheat-sheet/ (Accessed August 14, 2021)

https://medium.com/@ipspecialist/how-blockchain-technology-works-e6109c033034 (Accessed August 14, 2021)

$$
---X X X---
$$

